XIX.

Arbeiten aus dem Laboratorium für Physiologie zu Turin.

\title{
Die Lehre vom Fieber in Bezug auf die cerebralen Wärmecentren.
}

Studie über die Wirkung der Antipyretica.

\author{
Von \\ Dr. Ugolino Mosso. \\ (Mit 1 Abbildung.) \\ ERSTES KAPITEL.
}

Temperaturveränderungen nach Verletzungen des Gehirns beim Hunde.

Um den Mechanismus der Wirkung einiger zur Bekämpfung des Fiebers angewandter Arzneimittel $\mathrm{zu}$ studiren, ersehien es mir von Nutzen, zunächst festzustellen, ob im Gehirn wirklich Centren existiren, welche unter gewissen Umständen eine Steigerung der Körpertemperatur veranlassen, und ob die antipyretischen Mittel eine Wirkung auf diese Centren ausüben. Bekannt ist die in jüngster Zeit von Ch. Richet, Aronsohn und Sachs, Girard, Isaae Ott und Sawad owski aufgestellte Lehre, nach welcher sich im Gehirn des Kaninchens specielle Wärmecentren finden, deren Verletzung eine Erhöhung der Körpertemperatur zur Folge hat.

Ueber den Sitz der thermischen Centren im Gehirn des Hundes sind bis jetzt noch gar keine methodischen Untersuchungen angestellt worden. Durch eine Reihe von Vorversuchen habe ich mich überzeugt, dass sich zum Studium des Fiebers das Kaninchen von allen Thieren am wenigsten eignet, weil es schon unter normalen Verhältnissen eine sehr veränderliche Temperatur besitzt. Ich will die zahlreichen Daten, welche über diesen Punkt von mehreren Autoren, zuletzt von $\mathrm{C} . \mathrm{Golg} \mathrm{i}^{1}$ ), veröffentlicht worden sind, hier nicht anfübren, erwähnen möchte ich nur die Thatsache, dass in einem Versuche, wo Kaninchen sich in einem Wärmekasten von $35^{\circ}$ befanden, einige der Thiere in einem Zeitraum von $3{ }^{1 / 2}$ Stunden eine Temperaturerhöhung von $7^{0}$ aufwiesen. 
Wie die Temperatur, so ist auch die Frequenz der Herzaction des Kaninchens grossen Schwankungen unterworfen; schon Bunge hatte bemerkt, dass nach der Injection jeder beliebigen Substanz beim Kaninchen eine Steigerung der Pulsfrequenz eintritt.

Meine Experimente wurden an Hunden angestellt. Nach der Trepanation des Schädels bediente ich mich zur Punctur oder zur partiellen Zerstörung des Gehirns einer Nadel, ähnlich derjenigen, wie sie Cl. Bernard in seinen Versuchen am Boden des 4 . Ventrikels angewandt hat, oder eines Drahtes von $2 \mathrm{~mm}$ Durchmesser, der wie eine gewöhnliche Unterbindungsnadel leicht gebogen war. Dieses Instrument fithrte ich in die Gehirnsubstanz ein und drehte es, indem ich die Richtung des Stieles beibebielt, um seine Längsaxe, so dass eine intensive Verletzung des Gehirns an einem ganz bestimmten Punkte resultirte.

Zur Erzeugung ausgedehnter Läsionen benutzte ich einen Glasstab von $5 \mathrm{~mm}$ Dicke. Die Verletzungen, welche ich dem Gehirn beibrachte, betreffen hauptsächlich die Rinde, das Corpus striatum und den Thalamus opticus. Es mögen diejenigen Experimente den Anfang machen, in welchen die Grosshirnganglien unverletzt blieben.

Obgleich die Körpertemperatur des Hundes bedeutend geringeren Schwankungen unterliegt, als die des Kaninchens, so muss ich doch darauf aufmerksam machen, dass man auch hier in der Würdigung. der Thatsachen sehr vorsichtig sein muss, um nicht in Irrthümer zu verfallen. Man hüte sich davor, einer Temperaturerhöhung, wenn dieselbe nicht constant ist, irgend welehen Werth beizumessen, denn vorübergehende, oft sehr beträchtliche Schwankungen beobachtet man zuweilen als das Resultat einfacher psychischer Vorgänge, wie ich ${ }^{2}$ ) dies schon früher gezeigt habe. Meine damaligen Beobachtungen finden einen neuen Beleg in einem Experimente, in welchem ich ein continuirliches Ansteigen der Temperatur noch vor dem Beginn der Trepanation beobachtete; dieses Ansteigen der Temperatur dauerte fort, obwobl nach Beibringung der Gehirnläsion $5 \mathrm{~g}$ Chloralhydrat in die Bauchhöhle injicirt wurden. Zuletzt starb das Thier mit einer Temperatur von $43,15^{\circ}$, nachdem dieselbe in 1 Stunde 33 Minuten um $4,10^{\circ}$ gestiegen war, von denen allein $2,29^{\circ}$ anf die 30 Minuten vor Zufügung der Gehirnverletzung entfallen. Die Schnelligkeit der Temperatursteigerung, welche in diesem Experimente beobachtet wurde, ist überrasehend and könnte zu Irrthümern Veranlassung geben, wenn man nicht berticksichtigt, dass der Hund schon vor Eintritt der Rindenverletzung mehr als die Hälfte des Gesammt- 
zuwachses erreicht hatte und dass nach der Verletzung zum $\mathrm{Zu}$ standekommen einer geringeren Temperatursteigerung die doppelte Zeit nöthig war. Wir werden unten in $\$ 5$ sehen, dass nach ganz. ähnlichen Läsionen durchaus keine Erhöhung der Temperatur constatirt werden konnte.

Um die Wärmecentren zu suchen, habe ich 42 Versuche mit Trepanation des Schädels am Hunde gemacht. Die Beschreibung: dieser Experimente findet sich in meiner Abhandlung, welche von der medicinischen Akademie von Turin in diesem Jahre sublicirt. wurde. Hier gebe ich nur die Resultate.

§ 1. Läsionen der Gehirnrinde. In meinen Versuchen erbielt ich bei oberflächlichen Verletzungen in verschiedenen Regionen der Gehirnrinde bei Hunden keine beständige Temperaturveränderung. Bringt. man jedoch eine ausgedehntere Verletzung der Rinde in den vorderen Theilen des Grosshirns, wobei die Thalami optici und dje Corpora striata vollständig intact bleiben, hervor, so beobachtet man. ein beträchtliches Steigen der Temperatur.

Ein ähnliches Resultat erhält man bei Verletzungen, welche in der weissen Substanz der Occipitalwindungen gesetzt werden. Ich bediente mich zu diesem Zwecke eines Glasstabes von $5 \mathrm{~mm}$ Durchmesser, welchen ich in die hinteren Theile des Grosshirns, wo ja. die weisse Substanz die grösste Dicke besitzt, einbobrte.

Die Steigerung der Temperatur um 1,30, welche ich unter diesen Versuchsbedingungen erhielt, zwingt uns nicht zu der Annahme, dass. ein Wärmecentrum verletzt sei, weil ähnliche Steigerungen auch bei einfacher Trepanation beobachtet werden, und weil jene Temperaturerhöhnngen nach dem Losbinden des Thieres in einigen Stunden wieder rückgängig werden. Ein klares Beispiel hierfür finden wir in einem Experimente, in welchem die Temperatur, die unter der Einwirkung der Trepanation, der Erregung und des Schmerzes von $39,30^{\circ}$ auf $40^{\circ}$ gestiegen war, in weniger als $1 \frac{1 / 2}{2}$ Stunden um $1,5^{\circ}$ abfällt, obgleich die weisse Substanz in beträchtlicher Ausdehnung: zerstört worden war.

Die Resultate dieser ersten Reihe von Versuchen liefern den Beweis, dass bei Hunden die Verletzungen der Gehirnrinde keinen Einfluss auf die Temperatur des Mastdarms haben. Auf die Schlüsse, welche Wood und Richet aus ihren an Hunden angestellten Untersuchungen gezogen haben, werde ich bald zuriickkommen. Bis jetzt muss festgestellt werden, dass bei operirten Hunden die Temperatur sehr beträchtlichen Schwankungen unterliegt, und wir dürfen uns daher durch die nach Läsionen der Gehirnsubstanz auftretenden Ver- 
änderungen nicht zu leicht zur Annahme von thermischen Centren verleiten lassen. Hiervon habe ich mich auch durch die Beobachtung der operirten Hunde überzeugt, bei welchen ich die Temperatur in der Folge auf ihre normale Höhe zurïckkehren sah.

\& 2. Verletzung der Corpora striata und der Linsenkerne. A r onsohn und Sachs geben an, dass die stärksten Temperaturerhöhungen beim Kaninchen infolge von Verletzungen auftreten, welche die Corpora striata und die darunter liegenden Gehirntheile bis zur Basis des Craniums betreffen. Beim Hunde habe ich diese starken Temperatursteigerungen nicht beobachtet. Meine Experimente zeigen, dass eine Verletzung, welche auf das Corpus striatum und den Linsenkern einer Seite beschränkt ist, nur eine geringe Steigerung zur Folge hat, und dass dieselbe im Verlauf von 7 Stunden wieder verschwindet, selbst wenn die Verletzung der betreffenden Centren eine sehr tiefgreifende war.

Dieselbe Erhöhung der Temperatur tritt ein, wenn die Corpora striata und die Linsenkerne beider Hemisphären gleichzeitig und, ohne dass ein Bluterguss in die Seitenventrikel erfolgt, eine ausgedehnte Verletzung erfahren. Der Verlauf der Temperatur bei Verletzungen der Corpora striata und der Linsenkerne wies in meinen Experimenten nur geringe Verschiedenheiten auf. In 2 Versuchen blieb die Temperatur einige Stunden lang auf $39,5^{\circ}$ und sank dann unter $39^{\circ}$. In 2 anderen, welche durch Bluterguss in die Seitenventrikel complicirt waren, erreichte die Temperatur $40^{\circ}$ und blieb einige Zeit auf dieser Höhe, um dann wieder auf $39^{\circ}$ abzufallen. Wir können daher auf Grund dieser Beobachtungen nicht behaupten, Organe verletzt zu haben, welche der Wärmebildung des Körpers vorstehen. In einem anderen Experimente suchte ich dem Gehirn eine noch grössere Verletzung beizufügen, indem ich den ganzen mittleren Theil des Gehirns in der Höhe der Corpora striata zerstörte. Ich erhielt eine Erhöhung der rectalen Temperatur um etwa 2", welche jedoch weniger als 1 Stunde anhielt und darauf allmählich bis unter die normale Höhe abfiel.

Wenn wir noch einmal die Resultate der mitgetheilten Beobachtungen ins Auge fassen, so sehen wir, dass man beim Hunde die Corpora striata und die Linsenkerne in grosser Ausdehnung verletzen kann, ohne jene Temperatursteigerungen zu erhalten, welche als charakteristisch für die Läsionen dieser Hirntheile beim Kaninchen angesehen werden. Dass in den Corpora striata nicht der Sitz eines wärmebildenden Centrums zu suchen ist, dafür lassen sich auch noch andere Beweise beibringen; ich will nur e in en anführen, der mir sebr 
iuberzeugend erscheint. Bei einem Hunde zerstöre ich die Corpora striata und die Linsenkerne beider Hemisphären und erbalte eine Temperatursteigerung von über $1{ }^{10}$, welche etwa 1 Stunde anbält. Nachdem die Temperatur wieder auf $38,5^{\circ}$ gefallen ist, mache ich einen Aderlass und es erfolgt, wie vorher zufolge der Verletzung, eine Erhöhung der Temperatur um 1,50.

$\S 3$. Verletzungen des Thalamus opticus. Die Verletzungen des Thalamus opticus ergaben für die Theorie der Wärmecentren keine besseren Resultate. Bei allen Hunden war 3-4 Stunden nach der Verletzung eine Temperaturerhöhung von $1-2^{0}$ vorhanden und wie in den früheren Experimenten kehrte die Temperatur bald zur Norm zurück.

In 2 Versuchen waren die Thalami optici sehr schwer verletzt, und obgleich Hämorrhagien eintraten und die Hunde wäbrend der Beobachtungen eine ungewöhnliche Aufregung zeigten, fiel doch die Temperatur ganz allmählich $a b$ und hatte am nächsten Morgen die normale Höhe wieder erreicht. Der Temperaturabfall war noch grösser in einem Versuche, in dem ich die betreffenden Theile in grosser Ausdehnung verletzt hatte.

$\S 4$. Gleichzeitige Verletzung der Corpora striata, der Linsenkerne und der Sehhügel. In diesen Versuchen, welche ich an 5 Hunden anstellte, wurde als Maximum der Temperatur 40,80 bei einem Hunde beobachtet, bei dem sich in der Autopsie an der Basis des Gehirns eine die Nervenstämme umhüllende fibrinöse Masse fand.

$\S 5$. Versuche, in denen nach Gehirnläsionen eine Temperaturerhöhung beobachtet wurde. Es ist bekannt, dass Thiere derselben Species in den verschiedenen Lebensaltern oder bei verschiedenem Körpergewicht gewisse Differenzen in Bezug anf die Grösse des Stoffwechsels and die Wärmeproduction aufweisen. Um die Resultate meiner Untersuchungen getreu wiederzugeben, muss ich jetzt iuber diejenigen Versuche berichten, in denen ich zufolge verschiedener Gehirnläsionen eine Erhöhung der Temperatur eintreten sah. Es handelt sich hierbei um ganz abnorme Fälle, welche sehr verwirrend wirken können, wenn nicht die Untersuchung sich auf eine grosse Anzahl von Versuchsthieren erstreckt. Unter den 42 Hunden, die mir $\mathrm{zu}$ diesen Untersuchungen dienten, beobachtete ich nur bei 4 diese abnorme Temperatursteigerung, und zwar nach verschiedenen Verletzungen des Gehirns.

Ich hatte den Eindruck, dass diese Thiere sich sehr stark bewegten; ausserdem waren 3 derselben schon sehr alt. 
In einem der Experimente stieg die Temperatur trotz fortdauernder Chloroforminhalation nur, so lange der Hund aufgebunden war, und begann abzusinken, sowie der Glasstab aus dem Gehirn entfernt und der Hund in Freiheit gesetzt wurde. Diese Temperaturerhöhung war nicht durch eine Verletzung der regulatorischen Centren hervorgebracht, denn schon vor der Verletzung war die Temperatur in 42 Minuten um $0,91^{\circ}$ gestiegen und erhöhte sich in den nächsten 40 Minuten nach der Verletzung um 1,050, in den folgenden 50 Minuten um $0,92^{\circ}$. Der rasche Temperaturabfall nach dem Losbinden des Hundes beweist zur Genüge, dass die Steigerung der Temperatur nicht durch die Zerstörung eines regulatorisehen Centrums veranlasst, sondern die Folge einer psychischen Erregung. Dieselben Verhältnisse finden sich in einem anderen Versuche, in welchem ich, da mit Chloroform keine gute Narkose zu erzielen war, das Curare anwandte, um die Bewegungen des Thieres aufzubeben. Der aufsteigende Verlauf der Temperatur blieb unverändert von dem Momente, wo der Hund aufgebunden wurde bis zu seinem Tode, welcher nach der zweiten Curareinjection erfolgte.

\section{ZWEITES KAPITEL.}

\section{Die Lehre von den Wärmecentren.}

\$6. Tscheschichin ${ }^{3}$ ) war der Erste, welcher 1866 bein seinen Versuchen am Kaninchen, welche er im Laboratorium von Du BoisReymond ansführte, die Thatsache beobachtete, dass nach der Durchschneidnng des verlängerten Markes bei dessen Durchschnitt durch den Pons Varoli die Temperatur des Kaninchens zu steigen begann, und dass auch die Reflexerregbarkeit sich erhöhte. Nach 5 $1 / 2$ Stunden starb das Thier unter allgemeinen Krämpfen mit einer Temperaturerhöhung von $3,2^{0}$. Tscheschichin hat bei seinem Kaninchen keine Autopsie gemacht. 3 Jahre später konnte Lewizky ${ }^{4}$ ) an Kaninchen durch dieselbe Verletzung niemals solche starke Temperaturerhöhungen erhalten. Lewizky führt daher in seiner Kritik des Tscheschichin'schen Versuches die Steigerung der Temperatur auf die Convulsionen, die Muskelarbeit, zurïck. Ich habe mich bereits früher 2 a) üiber die Frage ausgesprochen, ob die Bildung von Wärme der Contraction der Muskeln zu verdanken, oder ob sie nicht eine Begleiterscheinung sei; ich glaube, dass die von Tscheschichin beobachtete Vermehrung der Körperwärme einer Reizung der Medulla oblongata zuzuschreiben ist. Indem wir jede Discussion bei Seite lassen, ist für uns allein das interessant, dass $\mathrm{Le}$ wizky das Experiment Tscheschichin's nicht bestätigt hat. R. Heidenhain ${ }^{5}$ ) veranlasste im folgenden Jahre zwei seiner Schüler, Bruck and $G$ ì $n$ ter, die einander widersprechenden Versuche von Tscheschi chin und Lewizky zu wiederholen. Von den 23 Versuchen, welche 
sie an Kaninchen anstellten, lieferten 11 ein entschieden positives, 12 ein negatives Resultat. Infolge dieser unsicheren Befunde versuchten B ruck und Gü ter, ob andere Gehirntheile constantere Resultate liefern würden. Nach Heidenhain, welcher die Versuche seiner beiden Schiler veröffentlichte, hat die einfache Punctur des Gehirns einen sichereren Erfolg, als die Durchschneidung, und wiederholte Stichelungen bringen immer wieder eine neue Temperatursteigerung hervor. Dies ist ein Beweis dafür, dass es sich um eine tranmatisclie Reizung umschriebener Gehirntheile handelt.

Die Versuche hatten das Verdienst, dem Problem eine neue Richtung gegeben zu haben; an Stelle einer Paralyse der moderirenden Centren wäre es nunmehr die Reizung der nervösen Centren, auf welche die Temperaturerhöhung zurückzuführen ist. Im Jahre 1874 veröffentlichte $\mathrm{Schreiber}{ }^{6}$ ) in seiner Inaugural-Dissertation eine Reihe von Versuchen am Kaninchen, welche er in der Klinik von $\mathrm{Naunyn}$ in Königsberg angestellt hatte. Er fand, dass die Läsionen des Pons, der Pedunculi und der dem Pons benachbarten Theile des Kleinhirns nur dann eine Temperaturerhöhung hervorbrachten, wenn die Thiere durch Einhüllen in Baumwolle oder Flanell oder durch eine künstlich erwärmte Umgebung vor Wärmeverlust geschützt wurden, und dass die Temperatur fiel, wenn die Wärmeabgabe nicht gehindert war. Er fand ferner, dass die Temperaturerhöhung constant und bedingungslos eintrat, wenn die Läsion auf die Grenze zwischen Medulla oblongata und Pons fiel; Durchschneidungen der Brücke in verschiedener Höhe hatten stets Temperaturerniedrigung zur Folge. Schreiber wollte sich iiber den Werth seiner Versuche nicht aussprechen und sagt nicht, ob sie die Lehre von den moderirenden oder excitatorischen Centren bereicherten, oder ob es sich einzig und allein um vasomotorische Phänomene handle. Trotzdem sind seine Resultate für uns werthvoll, weil auf dieselben die vasomotorischen Centren und das Centrum der Respiration nicht ohne Einfluss sein konnten, welche ja den verletzten Localitäten, besonders in dem kleinen Kaninchenhirn, so nahe liegen.

6 Jahre später veröffentlichte $W_{\text {ood }}{ }^{7}$ ) zahlreiche Experimente, die ersten, welche an Hunden angestellt waren. Die Verletzungen, welche er am unteren Rand der Brücke und am oberen Rand der Medulla oblongata erzeugte, gaben ihm stets eine Erhöhung der Temperatur, und Wood nimmt an, dass diese Temperaturerhöhung die Folge des Wegfalls eines moderirenden Centrums sei. Mit den Versuchen von $\mathrm{W}$ ood sind wir zum Ausgangspunkt, zur Hypothese Ts cheschichin's, zuriickgekehrt, obgleich Heidenhain die Untersuchung in richtige Bahnen gelenkt hatte. Indessen hatte Wood in der Frage der Localisation der Wärmecentren einen Schritt vorwärts gethan. Er hatte constatirt, dass die Störungen der Hitzig'schen Region Temperaturerböhungen hervorbrachten, wie sie die übrigen Theile der Gebirnrinde nicht geliefert hatten. Er hatte jedoch so wenig Zutrauen zu den Ergebnissen seiner Versuche, dass er erklärt, diese Region sei nicht der eigentliche Sitz des Wärmecentrums, sondern stehe zu diesem nur in einem gewissen Zusammenhang, so dass der Zustand dieser Region auf den Zustand des hypothetischen Centrums von Einfluss sei. Es war somit den Untersuchungen über den Sitz der regulatorischen Centren der Körperwärme ein nenes Feld eröffnet. 
Ch. Richet $^{8}$ ) stichelte mit einer stählernen Nadel die Vorderlappen des Kaninchenhirns unter Schonung der Corpora opto-striata und erhielt in 3 Fällen eine Erhöhung der Temperatur von 1,1--3,4\%. Ein 4. Kaninchen, welches in der Nacht starb, zeigte die stärkste Erhebung um $3,6^{\circ}$ in 45 Minuten. Die Versuche von $\mathrm{Ch}$. Richet haben nicht den Nachweis geliefert, dass im Gehirn des Kaninchens Theile existiren, deren Läsion in constanter Weise eine Temperaturerhöhung zur Folge hat, geschweige denn, dass sie über den Sitz dieser Theile einen Aufschluss geben könnten. Gleichzeitig mit $\mathrm{Ch}$. Richet veröffentlichte Isaae 0 tt $^{9}$ ) seine calorimetrischen Versuche und gab an, dass in der Nähe der Corpora striata des Kaninchens ein Centrum existire, welches einen Einfluss anf die Temperatur habe; doch konnte er sich nicht dafür entscheiden, ob es sich um eine vermehrte Wärmeproduction oder um eine verminderte Wärmeabgabe handle. In demselben Jahre, 1885, führten Aronsohn und Sachs ${ }^{10}$ ), ohne von den Beobachtungen Ch. Richet's Kenntniss zu haben, im Laboratorium von Kronecker in Berlin methodische Stichelungen, Kauterisationen und Excisionen in den verschiedenen Theilen des Kaninchenhirns aus und fanden, dass die mediale Portion der Corpora striata und der darunterliegenden Theile bis zur Basis die einzige Region sei, deren mechanische oder elektrische Reizung einen Einfluss auf die thierische Wärme, und zwar im Sinne der Erhöhung, ausübe. Allein die Temperaturerhöhungen, welche A ronsohn und Sachs erhielten, treten verspätet ein (24-70 Stunden nach der Stichelung), bleiben nur kurze Zeit constant und das Kaninchen kehrt nach kürzerer oder längerer Zeit zu seiner ursprünglichen Temperatur zurück.

Es kommen hierauf die Versuche von Girard 11) welche im physiologischen Laboratorium zu Genf, gleichfalls an Kaninchen und ohne eine Vorrichtung zur Verhinderung der Abkühlung angestellt wurden. Girard bestätigte in dieser seiner ersten Arbeit die Schlüsse von Aronsohn und Sachs, jedoch mit einigen. Einschränkungen in Bezug auf Grösse und Dauer der Temperaturerhöhung. Die calorimetrischen Experimente von Isaac $0 \mathrm{tt}^{12}$ ) 1887-1888 führten ihn dazu, 4 Wärmecentren aufzustellen, eines vor und unter dem Corpus striatum und dem Linsenkern, ein anderes an der Convexität des Nucleus caudatus, ein drittes in der Laminia (Stria) cornea, ein viertes im medialen vorderen Theil des Thalamus opticus. Girard (3) kommt in seinem letzten Werke, auf Grund zahlreicher Versuche, die er alle an Kaninchen anstellte, za dem Schlusse, dass die Gehirnregionen, welche physiologischerweise der Wärmebildung vorstehen, offenbar sehr zahlreich sind, und dass die Regulirung der Wärmeproduction im Organismus einem sehr complicirten centralen Apparate zu verdanken sei. Ich begrïsse diesen Schluss von Girard mit Freude, weil er vollständig das bestätigt, was ich in Bezug auf die Wärmecentren in meiner ersten Arbeit, die im Giornale della $R$. Accademia di medicina di Torino erschienen ist, seit dem Jahr 1885 ausgesprochen habe.*)

*) Ich bin überzeugt, dass das sogenannte regulirende Wärmecentrum dasselbe Schicksal haben wird wie die vasomotorischen Centren und dasjenige der Athmung, und wenn solche Centren existiren, so müssen sie nicht nur im Gehirn, 
Die Versuche von Goltz über das Gehirn des Hundes, in denen er einen grossen Theil der Rindensubstanz abträgt, ohne dass eine dauernde Alteration der Körpertemperatur beobachtet wird, und die von Corin und van Beneden, welche einen grossen Theil des Gehirns entfernten, führen uns zur Ueberzengung, dass es im Gehirn keine Centren giebt, denen ausschliesslich die Regulirung der Wärme des Organismus obliegt.

Bei den Versuchen, welche ich selbst am Kaninchenhirn angestellt habe, erbielt ich bei Läsionen, die nur die Rinde betrafen, keine Temperaturerhöhung, die Verletzungen der Nuclei gaben mir blos vorübergehende Steigerungen; die starken Temperaturerniedrigungen waren stets von schweren Verletzungen tieferer Gehirntheile begleitet, was ich auch bei Hunden beobachtete, doch habe ich in meinen Versuchen an Kaninchen und Hunden einige Thatsachen feststellen können, welche mir die Annahme im Gehirn localisirter thermischer Centren unthunlich erscheinen lassen:

I. Die Temperaturerhöhung nach Gehirnläsionen ist nie so gross, dass man nicht mittelst Cocaineinspritzungen aufs Neue eine beträchtliche Steigerung der Temperatur erhalten könnte. II. Die Verminderung der Temperatur nach Verletzungen des Gehirns hängt nicht von einer Läsion ab, welche die thermischen Centren lähmt, weil bei Anwendung von Cocain die Temperatur sobald wieder steigen kann.

Die Lehre von den thermischen Centren ruht, wie aus diesem kritischen Ueberblick hervorgeht; auf einer recht unsicheren Grundlage; bis jetzt haben wir keine zuverlässigen Experimente und keine anatomischen oder physiologischen Untersuchungen, welche uns über die Structur oder über die Function dieser Centren eine Aufklärung. versehafften. Selbst die Anhänger der Lehre von der Existenz der Wärmecentren können nicht angeben, ob sie mit den in den nervösen Centralorganen gesetzten Verletzungen Nervenfasern durchtrennt, Gruppen von Nervenzellen zerstört haben, welche die chemischen Processe in den Geweben beschleunigen, oder solche, welche dieselben verlangsamen. Ich hoffe den Beweis geliefert zu haben, dass im Gehirn des Hundes keine Centren vorhanden sind, nach deren Zerstörung constant eine Erhöhung oder Verminderung der Temperatur eintritt, und ich glaube, dass auch im Gehirn des Kaninchens diese regulatorischen Centren fehlen'. Die Autoren, welche dieselben angenommen haben, müssen ihre Experimente wieder anfnehmen, um zu zeigen, was diese Centren eigentlich sind; ob wir bei

sondern auch im Rückenmark, ja vielleicht überall da zu finden sein, wo eine Anhäufung von Nervenzellen vorhanden ist. 
den Gehirnläsionen Bahnen zerstören, welche das Gehirn zu entfernter liegenden Apparaten, von denen die Wärmebildung abbängt, in Beziehnng setzen, ob wir die Verbindung von Theilen aufheben, welche gegenseitig einen moderirenden Einfluss ausïben, oder ob im Gehirn die Strassen von weiter abliegenden Centren verlaufen.

Die Versuche, welche ich mit Strychnin und Cocain angestellt habe ${ }^{2}$ ), zeigen, mit welcher Schnelligkeit der thierische Organismus bedeutende Wärmemengen zu entwickeln im Stande ist. Auf S. 93 meiner Arbeit ${ }^{2}$ ) habe ich über ein Experiment berichtet, bei welchem ein bis zur Unbeweglichkeit curarisirter Hund seine Temperatur zufolge der Injection passender Strychnindosen in 2 Stunden und $40 \mathrm{Mi}$ nuten um $3^{0}$ erhöhte, und in einem anderen Versuche erhielt ich (S. 200 der zweiten Arbeit ${ }^{2 b}$ )) eine Erhöhung der rectalen Temperatur um $4,7^{\circ}$ in nur 45 Minuten nach der Darreichung von Cocain. Um die Existenz in irgend einem Theile des Nervensystems localisirter Wärmecentren behaupten zu können, muisste man durch die Reizung oder Zerstörung derselben ähnliche Temperaturerhöhungen erhalten.

Ich fürchte jedoch, dass wir diese Centren in keinem Theile des Nervensystems isolirt antreffen werden.

Um mich nicht darauf zu beschränken, meine Beobachtungen denen angesehener und erfahrener Experimentatoren einfach gegentiberzastellen, wollte ich versuchen, ob nicht trotzdem eine weniger exacte Auslegung der Thatsachen dazu hätte führen können, die Existenz von thermischen Centren anzunehmen. C. Richet beobachtete ganz richtig, dass die erhöhte Wärmebildung nach den Gehirnläsionen mit einer gesteigerten Erregbarkeit des Kaninchens gleichen Schritt bält. Ich lege dieser Thatsache ein grosses Gewicht bei, weil sie uns zur Lösung der Frage auf ein anderes Gebiet hinweist. Bei Kaninchen tritt die Erhöhung der Temperatur nach einer Gehirnläsionen nicht deshalb ein, weil diese oder jene Region verletat worden ist, sondern nur deshalb, weil das Thier schon von vornherein disponirt war, auf eine beliebige Reizung des Nervensystems mit intensiveren intracellulären chemischen Vorgängen zu reagiren. Meine Versuche an Hunden stimmen mit dieser Annahme überein. Die Hunde sind von Natur weniger erregbar als die Kaninchen, und so fand ich denn unter 42 Hunden nur 4, welche nach Verletzung des Gehirns eine Temperaturerhöhung darboten. 3 von diesen waren schon alte Thiere. Dass die Hyperthermie nicht von der Läsion eines speciellen Centrums abhängig ist, geht ohne Weiteres darans hervor, dass bei jenen Thieren ganz verschiedene Theile des Gehirns zerstört worden waren. Beweisend ist auch die Thatsache, dass 
ähnliche Erhöhungen auch eintreten, ohne dass eine Gehirnverletzung beigebracht wird. Ich fand einige alte Hunde, welche eine Erhöhung: der rectalen Temperatur um $2-3^{0}$ zeigten, einfach weil sie auf den Operationstisch aufgebunden worden waren. Die Temperaturerhöhung, welche man infolge einer Gehirnläsion beobachtet, würde demnach durch abnorme psychische Vorgänge, durch eine pathologische Exaltation und Erregung des Nervensystems zu erklären sei und nicht durch den mangelnden Einfluss eines thermischen Centrums.

\section{DRITTES KAPITEL.}

\section{Calorimeirische Messungen und Kritik derselben.}

$\S 7$. Beschreibung eines neuen Calorimeters. Da man gewöhnlich annimmt, dass die Kenntniss der Mastdarmtemperatur zu der Entscheidung, ob die Wärmeproduction vermehrt oder vermindert ist, nicht ausreiche, unterzog ich in einer Reihe von Versuchen die Wärmemengen, welche der Organismus durch die Lungen und die Haut verliert, einer quantitativen Bestimmung.

$\mathrm{Zu}$ diesem Zwecke construirte ich ein Calorimeter, welches in Fig. 1 wiedergegeben ist. Das Calorimeter ist ein grosser, sehr empfindlicher Luftthermometer, welcher die Veränderungen der Temperatur anzeigt. Derselbe beruht auf dem Princip der von D'Arsonval ${ }^{15}$ ), Richet ${ }^{16}$ ), Rosenthal ${ }^{17}$ ), Langlois ${ }^{18}$ ) und Frederic ${ }^{19}$ ) angewandten Instrumente und besteht aus zwei concentrischen Metalleylindern, welche mit Ausnahme der unten zu erwähnenden Oeffnungen hermetisch verschlossen sind. Der innere Recipient $\mathbf{F}$, der mit einer punctirten Linie in den grossen äusseren Recipienten eingezeichnet ist, nimmt das Versuchsthier auf und hat einen Durchmesser von 34 und eine Höhe von $24 \mathrm{~cm}$. Um ihn hermetisch zu verschliessen, bediene ich mich eines Deckels, auf dessen Peripherie ein Messingreif von $4 \mathrm{~mm}$ Dicke und $2 \mathrm{~cm}$ Höhe senkrecht aufgelöthet ist. Dieser Rand greift in eine Rinne von $6 \mathrm{~mm}$ Breite und $2 \mathrm{~cm}$ Tiefe, welche in den oberen Rand des cylindrischen Recipienten eingelassen ist. Der innere Cylinder hat 2 Oeffnungen, um dem Versuchsthier Luft zuzuführen. Die Röhren, welche seinen Hohlraum vermittelst der Röhren $\mathrm{G}$ und $\mathrm{H}$ mit der umgebenden Luft in Verbindung setzen, sind hell gezeichnet; die eine mündet am Dach, die andere am Boden der Kammer F. Der äussere Cylinder, gleichfalls von Messing, hat einen Durchmesser von $42 \mathrm{~cm}$, eine Tiefe von $33 \mathrm{~cm}$; in ihm ruht auf 3 Korkstiucken der innere Cylinder $F$, so dass zwischen den Wänden der beiden Cylinder ein mit den Buchstaben I, I, I bezeichneter Hohlraum bleibt. Der äussere Cylinder ist von einer (hier nicht gezeichneten) hölzernen Kammer umgeben, um den Einfluss der Sehwankungen der Zimmertemperatur auszuschliessen.

In dem Maasse, als das Thier den Luftraum I, I, I zwischen den beiden Cylindern erwärmt, entweicht die Luft durch die Röhre L, tritt in 
Die Lehre rom Fieber in Bezug auf die cerebralen Wärmecentren. 327 die Woulf'sche Flasche ein und drängt das Wasser aus derselben durch die Röhre M, M in den schwimmenden Cylinder $\mathrm{N}$ des Plethysmographen.

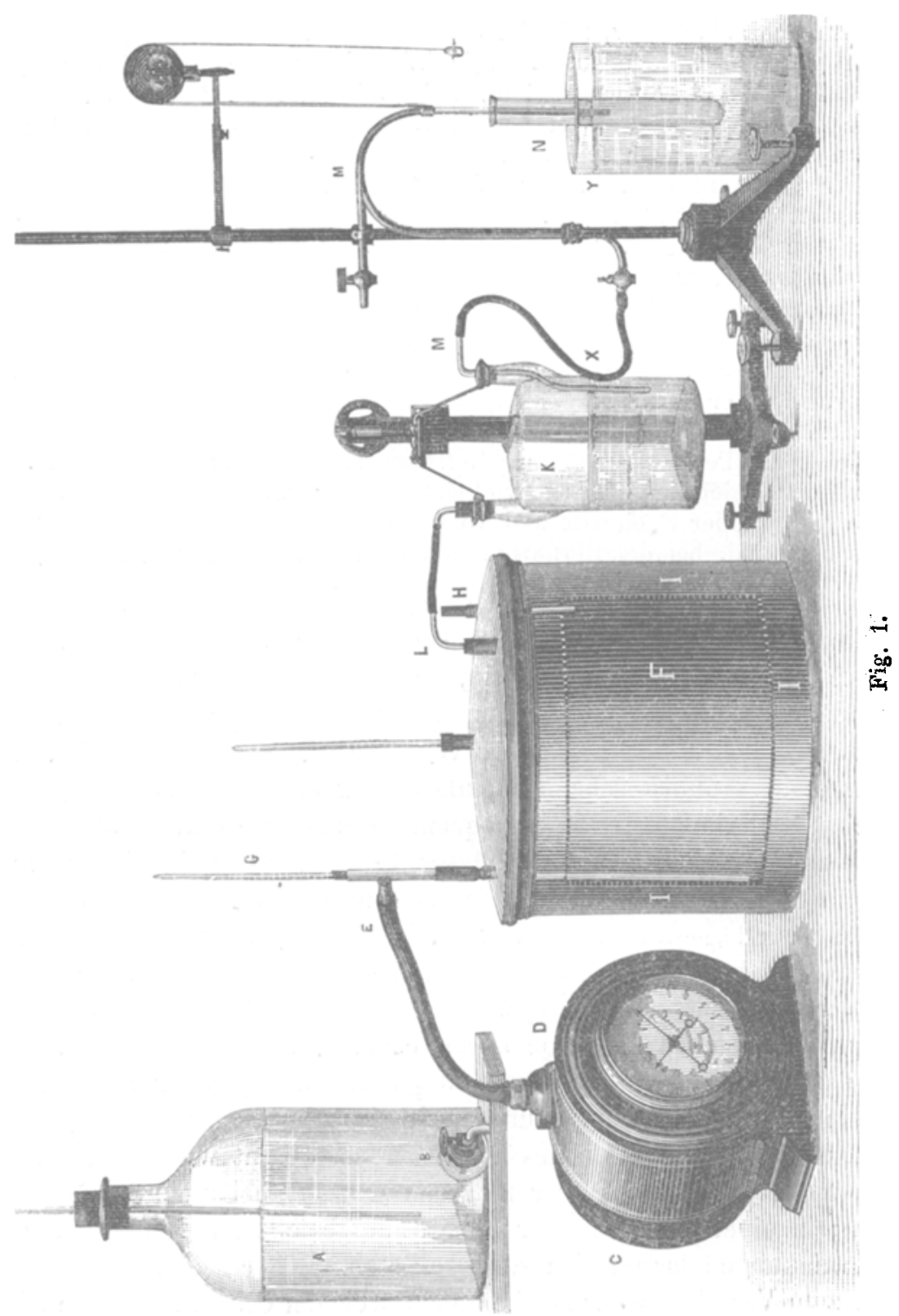

Mit der Schilderung dieses Apparates brauche ich mich nicht aufzuhalten, da derselbe bereits von meinem Bruder beschrieben ist ${ }^{14}$ ). Um das A rch i v f, experiment. Pathol. u. Pharmakol, XXVI, Ba. 
Niveau $X$ des Wassers in der Woulf'sehen Flasche inmer mit dem Niveatu $Y$ der Wasseralkoholmischung des PTethysmographen auf gleicher Höhe erhalten zu können, befestigte ich die Woulf'sche Flasche $K$ an einem Schraubenstativ, mittelst dessen sie mit Leichtigkeit ganz allmählich gehoben werden kann. Die Woulf'sche Flasche und der grosse Behälter des Plethysmographen, welche ich in Fig. 1 weit auseinander zeichnen musste, berühren sich in Wirklichkeit, so dass die beiden $\mathrm{Ni}$ veau $X$ und $Y$ sehr leicht in Uebereinstimmung zu erhalten sind und daher der Luftdruck in der Woulf'schen Flasche und im Calorimeter constant gleich Null bleibt.

Um die Luft im Recipienten $F$ zu erneuern, bediene ich mich eines Aspirators D, welcher die Menge der nach F eintretenden Luft misst. Ein kleines nach Art der Wassermühlen construirtes Cassettenrad ist an die Axe des Zählapparats angebracht. Aus einer grossen Mariotte'schen Flasche A erbält man dürch den Hahn B einen constanten Wasserausfluss, der dem Rad des Zählapparates die gewïnschte Geschwindigkeit verleiht. In einer Oeffnung des Deckels befindet sich ein Thermometer, welcher die Temperatur in dem Luftraum I, I, I misst; ein anderes Thermometer G giebt an, um wie viel sich die Luft, welche durch die Röhre II in den Cylinder $\mathrm{F}$ eintritt und vom Zählapparat durch die Röhre $\mathrm{E}$ angesangt wird, bei der Berührung mit dem Versuchsthier erwärmt hat.

Ichi glaube, dass mein Calorimeter gegentiber den von anderen Autoren angewandten Calorimetern gewisse Vorzüge besitzt. Eine erste Verbesserung besteht darin, dass man nicht nur die Erhöhungen, sondern auch die Verminderungen der Temperatur registriren kann, was mit dem Hebercalorimeter von Richet nicht möglich ist. Auf den ersten Anblick scheint es, als ob der Verschluss der Recipienten meines Calorimeters weniger bequem zu handhaben wäre, als bei den anderen Apparaten, bei genauerer Prüfung stellt sich jedoch mein Verfahren als praktischer heraus. Bei den anderen Calorimetern muss man 1/1/2--2 Stunden warten, bis sich die Luft des Calorimeters abgekühlt hat, welche meistens zwischen metallischen Wänden oder in einer gewundenen Röhre, wie in dem Ei von Richet, in den Brütapparaten, im Apparat von d'Arsonval, eingeschlossen ist. Mein Apparat dagegen erlangt, wenn die einzelnen Cylinder auseinandergenommen sind, in ganz kurzer Zeit die Temperatur der Umgebung. Um naeh der Operation des Thieres den Apparat wieder zusammenzusetzen, bedarf es keiner längeren Zeit als bei anderen Calorimetern; in einer Minute ist der Apparat aufgestellt und die Temperaturschwankungen lassen sich sofort ablesen. Ich erreichte dies, indem ich zum Verschluss der Deckel recht weichen Glaserkitt in die Rinne am oberen Rand der beiden Cylinder einstrich. Da in dem Hohlraum zwischen den beiden Recipienten kein von dem àtmosphürischen abweichender Drtick herrschen soll, sơ ist đies đie einfachste Methode, 
um einen luftdichten Abschluss herzustellen, ohne sich unangenehmen Zwischenfällen auszusetzen, welche sich bei der Anwendung von Wasser oder Quecksilber nicht ganz vermeiden lassen.

Eine weitere Verbesserung, welche ich bei meinem Calorimeter einführte, ist die Circulation der von dem Thiere geathmeten Luft. Die Aspiration, welche durch die gleichförmige Bewegung des Zählapparates D erfolgt, erlaubt die vom Thier ausgeathmete Kohlensäure und das Wasser durch geeignete, mit der Röhre $\mathrm{E}$ in Verbindung gesetzte Apparate zu bestimmen. D'A rs onval bewerkstelligte die Ventilation durch den Luftzug einer Flamme, welche in einer mit dem Apparat verbundenen Röhre angezündet wurde. Bei meinem Calorimeter ist es leicht, den richtigen Grad der Lüftung hervorzubringen, welche die Kohlensäure und den Wasserdampf entfernt, ohne einen zu starken Luftzug zu erzeugen. So vermeidet man die unangenehmen Folgen, welche die Anhäufung der Kohlensäure haben könnte, und wenn nach einigen Stunden das Thier herausgenommen wird, so zeigt sich sowohl dieses, als auch die Wand des Recipienten trocken.

Aus leicht begreiflichen Gründen muss man die Woulf'sche Flasche und den Behälter des Plethysmographen recht gross nehmen. Zur Ausführung dieser Versuche empfiehlt sich am meisten ein nach Norden gelegenes und geräumiges Zimmer, so dass die Schwankungen der Zimmertemperatur selbst bei der Anwesenheit des Experimentators auf ein Minimum reducirt werden. Beispielsweise führe ich an,

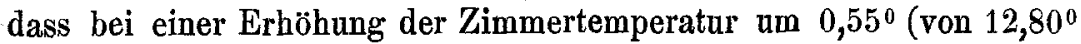
auf $13,35^{\circ}$ ), welche sich von $8 \mathrm{Uhr} 25$ Minuten Morgens bis Abends 5 Uhr 40 Min. einstellte, eine Erwärmung des Luftraums I am $0,5^{\circ}$ und eine Ausdehnung desselben um $34 \mathrm{ccm}$ erfolgte. Da nun dieser Luftraum $21950 \mathrm{ccm}$ misst und die Erwärmung desselben um $0,5^{0}$ nach der Berechnung eine Ausdehnung um $39,5 \mathrm{ccm}$ zur Folge haben missste, so kann man nach der Uebereinstimmung, in weleher sich die Resultate der Beobachtung und der Berechnung befinden, wohl behaupten, dass dieses Calorimeter die Ausdehnung der Luft mit hinreichender Genauigkeit misst. Da es sich jedoch nicht um die Messung der wirklichen Werthe der Temperaturschwankangen, sondern nur um vergleichende Versuche handelt, so geben die mit meinem Calorimeter erhaltenen Werthe ein genaues Bild von dem Wärmeverlust des Versuchsthieres.

\$ 8. Kritik der an Thieren angestellten calorimetrischen Messungen. Um zu beweisen, dass bei den von mir am Gehirn operirten Thieren keine vermehrte Wärmebildung stattfindet, welche bei Messung 
der Mastdarmtemperatur etwa der Beobachtung entginge, weil sie durch einen grösseren Wärmeverlust an der Körperoberfläche compensirt würde, dient der Versuch mit einem Hunde, welcher schon mehrfach zu calorimetrischen Messungen verwendet worden war und der sich daher in den günstigsten Versuchsbedingungen befand. Es zeigte sich, dass der Hund nach der Verletzung der Thalami optici unter gleichbleibenden Bedingungen in 2 Stunden 45 Minuten eine Wärmemenge entwickelte, welche um 1/7 geringer ist, als die zuerst gebildete. Dieses Resultat stimmt mit den Messungen der Mastdarmtemperatur überein, denn in der grossen Mehrzahl der Fälle haben wir bald nach der Verletzung verschiedener Hirntheile eine kurzdauernde, mehr oder weniger ausgesprochene Erniedrigung der Mastdarmtemperatur beobachtet. Diese Verminderung der Wärme könnte auf einen vermehrten Wärmeverlust zurückgeführt werden, und das Ergebniss des Versuches ist deshalb von Bedeutung, weil es zeigt, dass nach der Operation sogar eine geringere Wärmeabgabe stattgefunden hat.

Durch wiederholte derartige Versuche kam ich zu der Ueberzeugung, dass die Schwankungen der Temperatur im Rectum nicht von einer grösseren oder geringeren Wärmeabgabe abhängen, sondern dass bei Hunden die Mastdarmtemperatur mit hinreichender Genauigkeit die Intensität der Wärmebildung im Organismus angiebt.

Die Wärmemenge, welche der Organismus beständig durch die Respiration verliert, ist so gross, dass im Vergleich mit ihr der Wärmeverlust an der Körperoberfläche, besonders bei dicht behaarten Thieren, gewiss ganz verschwindend ist. Auch die kleinste Veränderung in der Frequenz und Tiefe der Athembewegungen überwiegt in ihren Folgen bei Weitem jede Schwankung, welche durch Erweiterung oder Verengerung der Hautgefässe zu Stande kommen kann. Die Schwankungen der Bluttemperatur, welche von einer grösseren oder geringeren Wärmeabgabe durch die Lungen und die Haut abhängen könnten, sind demnach kleiner, als es zunächst den Anschein hat. Aber selbst, wenn bei einer Erhöhung der Mastdarmtemperatur gleichzeitig eine Verminderung der Wärmeabgabe beobachtet wäre, so würde es doch noch immer schwierig zu entscheiden sein, ob nicht trotzdem eine thatsächliche Erhöhung der Wärmeproduction stattgefunden habe.

Man müsste an die calorimetrische Messung noch die Bestimmung des verbrauchten Sauerstoffs anschliessen, und auch das wäre noch nicht hinreichend, da nach den Versuchen von Pettenk ofer und Voit die Bildung von $\mathrm{CO}_{2}$ und der Verbrauch von Sauerstoff 
sich nicht genau entsprechen. Thatsächlich giebt es gewisse Tageszeiten, in denen eine grössere Menge Sauerstoff verbraucht wird, ohne dass darum die 0xydationsprocesse vermehrt wären oder eine grössere Quantität Kohlensäure producirt würde. Während des Schlafes und bei vollkommener Ruhe absorbirt das Thier mehr Sanerstoff und scheidet weniger Kohlensäure aus.

Die Methode der Wärmemessung zeigt solche Complicationen, dass sie sich nicht so nützlich erweist, als man glauben sollte. Die Temperaturdifferenzen, auf deren Bestimmung es hier ankommt, sind derart, dass man sich auch ohne calorimetrische Messungen Gewissheit darïber verschaffen kann, ob nach einer Gehirnläsion eine grössere oder geringere Wärmeproduction stattfindet.

Mit Hülfe meines Calorimeters wollte ich untersuchen, ob die Temperatursteigerung beim Fieber einer grösseren Wärmeproduction oder einer verminderten Wärmeabgabe zu verdanken sei. Meine Beobachtungen führten mich zur Bestätigung dessen, was Ch. Richet vor Kurzem ausgesprochen hat, dass nämlich für das Mehr oder Weniger an Wärme im Organismus nicht der geringere oder grössere Wärmeverlust dureh Strahlung, sondern thatsächlich die grössere oder geringere Wärmeproduction in den Geweben bestimmend ist.

Es ist bekannt, dass die Pathologen zu der Annahme neigen, dass die Erhöhung der Temperatur im Körperinnern durch die Retention der in normaler Menge gebildeten Wärme zu Stande komme und nicht durch eine Vermehrung der Wärmebildung, obgleich deren Möglichkeit nicht geleugnet wird.

Ich stellte mehrere calorimetrische Versuche an fiebernden Thieren an und fand ohne Ausnahme, dass bei Erböhung der Mastdarmtemperatur auch die Wärmeabgabe von der Körperoberfläche eine grössere wurde. Ich kann daher nicht daran zweifeln, dass in den von mir beobachteten Fieberfällen eine vermehrte Wärmeproduction vorhanden war. Diejenigen Autoren, welche die febrile Temperatursteigerung auf eine einfache Wärmeretention zurïckführen, werden zugeben müssen, dass die Steigerung der chemischen Processe in den Geweben die eigentliche Ursache des Fiebers ist. Gewiss kommt die durch die Wirkung der Vasomotoren entstehende Herabsetzung der Wärmeabgabe hinzu, wie dies die schönen Versuche von Prof. E. Maragliano ${ }^{20}$ ) in seiner Studie über das Verhalten der Gefässe im Fieber darthun, aber es wäre eine Uebertreibung, wenn man das Fieber auf ein einfaches Gefässphänomen und auf eine Verminderung der Wärmestrahlung zurïckführen, wenn man den Einfluss der Antipyretica einfach der lähmenden Wirkung, welche diese Mittel 
auf die Blutgefässe und die vasomotorischen Nerven austiben können, zuschreiben wollte. Ich halte es dagegen für viel wahrseheinlicher, dass die gefässlähmende Wirkung der Fiebermittel stets mit einem herabsetzenden und moderirenden Einfluss auf die chemischen Processe in den Geweben verbunden ist.

Die Untersuchungen, welche an Thieren angestellt werden, befriedigen mich nicht, weil die calorimetrische Methode neue Fehlerquellen in den Versuch einführt, und oft veranlassen die Versuchsbedingungen Temperaturschwankungen, grösser als diejenigen, welche als Resultat des Experimentes gesucht werden. Bis jetzt müssen wir uns bei der Bestimmung der von den Thieren gebildeten Wärmemenge mit einer relativen Genauigkeit und mit verhältnissmässig: kurzen Beobachtungszeiten begnügen. Angenommen, dass sich die Versuchsthiere im Calorimeter ganze Tage hindurch unter normalen Bedingungen halten liessen, dass es nicht nöthig wäre, den Apparat häufig zu öffnen und abzukühlen und dass sich jede Ursache für eine psychische Störung vermeiden liesse, so bliebe noch übrig, die specifische Wärme der animalischen Gewebe genall zu bestimmen, um die Menge der erworbenen und der verlorenen Wärme unseres Körpers zu kennen, so bliebe noch übrig, die Temperatur des Thieres während seines Verweilens im Calorimeter zu messen und eine Methode zu finden, um den Zustand der Blutgefässe während des Versuchs zu beobachten. So lange nicht die Mittel gefunden sind, um allen diesen Bedingungen za genügen, werden wir bekennen müssen, dass die calorimetrische Methode zu unvollkommen ist, und dass die Messung der Mastdarmtemperatur bis jetzt die sicherste Methode ist, um die Intensität der chemischen Umsetzungen, von denen die Temperatur des Organismus abhängt, zu ermessen.

\section{VIERTES KAPITEL.}

\section{Die Lehre vom Fieber und die Wirkung der Antipyretica.}

\$ 9. Wirkung des Antipyrins auf die cerebralen Wärmecentren. Gegen Ende 1887 erschienen die ersten pharmakologischen Arbeiten, welche auf der Basis der neuen Theorie von den Wärmecentren die Wirkung der Antipyretica und die Natur des Fiebers zu erklären suchten. Es ist natürlich, dass man, wenn man die Existenz dieser Centren im Gehirn annahm, 1. die Erscheinungen des Fiebers anf functionelle Störungen derselben zurückzuführen und 2. zu beweisen suchte, dass die Antipyretica eine Wirkung auf diese Centren ausüben. 
Diesen Zweck verfolgen die Untersuchungen von Girard 21) über. die Wirkung des Antipyrins. Es wäre ihm gelungen, den Satz aufzustellen, dass das Antipyrin in wirksamer Weise die durch Reizung eines cerebralen Wärmecentrums hervorgerufene Temperatursteigerung bekämpft, doch spricht er sich sehr unsicher über die Resultate seiner Versuche mit Antipyrin aus. Gleiehzeitig mit Girard arbeitete $\mathrm{Sawadowski}{ }^{22}$ ) in Petersburg über die Antipyrinwirkung, mit ebenfalls unsicheren Resultaten*).

Die Versuche mit Funden, denen vor oder nach der Zufügung: einer Gehirnläsion Antipyrin beigebracht wurde, ergaben so unbedeutende Veränderungen der Temperatur, dass jede Schlussfolgerung verfrüht wäre. Nachdem ich mich versichert hatte, dass das Antipyrin, vor oder nach der Verletzung der Gehirncentren dargereicht, den Verlauf des Experimentes nicht wesentlich beeinflusste, und dass die Mastdarmtemperatur beinahe dieselben Schwankungen aufwies, welche ich im ersten Kapitel geschildert habe, unternahm ich es, die Wirkung des Antipyrins bei fiebernden Hunden zu studiren.

$\$$ 10. Das Fieber durch Blutentziehung und Wirkung der Medicamente anf dasselbe. Wenn ein Hund ungefähr die Hälfte seines Blutes oder anch nur $4 / 10$ desselben (unter der Annahme, dass das Gewicht des Blutes 1/12 des Körpergewichts beträgt) verliert, so kann die Temperatur im Rectum folgende Modificationen zeigen:

1. eine variable Vermehrung um wenige Zehntelgrade, oder aveh um mehr als einen Grad, welche während des Aderlasses eintritt. 2. An diese momentane Erhöhung, welcher zuweilen ein rascher Abfall

*) Saw a dowski beschreibt in folgendermaassen die Antipyrinwirkung: „Nimmt man also an, dass in den Corp. striat. die regulatorischen thermischen Nervencentren gelegen sind; so kann man auf Grund der geschilderten Erscheinungen annehmen, dass in dem vorderen Theile der Corp. striat. das vasomotorische, thermische Centrum der Hautgefässe gelegen ist, dagegen in dem hinteren Theile derselben der wärmeproducirende, sogenannte trophische Abschnitt dieses Centrums, auf den das Antipyrin ebenfalls einwirkt, sich befindet. Die Wirkung des Antipyrins kann somit auf folgende Weise dargestellt werden: erstens bewirkt dasselbe eine Steigerung der Wärmeabgabe, indem es das specielle vasomotorische, thermische Centrum reizt, welches wahrscheinlich in dem vorderen Theile der Corp. striat. gelegen ist; da das Antipyrin ferner auch dann die Temperatur herabsetzt, wenn aus irgend welchem Grunde (Entfernung des entsprechenden Centrums, Befinden des Thieres in einem Raume mit hoher Temperatur) die Wärmeabgabe nicht gesteigert ist, so wirkt dasselbe folglich auch auf den anderen, sogenannten trophischen Abschnitt des genannten Centrums, indem das Antipyrin wahrscheinlich eine Lähmang des wärmeproducirenden Theiles desselben hervorruft oder vielleicht die Wärmeproduction im Körper herabsetzt." Dieses Bruchstück giebt uns ein Bild von der Unsicherheit und von der Verwirrung, welche in diesem Gebiete herrscht. 
nachfolgt, schliesst sich eine zweite, welche langsam ansteigt und mehrere Stunden andauert, oft von Schüttelfrösten begleitet. 3. eine Temperatursteigerung, welche sich in den folgenden Tagen wie eine Febris continua einstellt and mitunter $40^{\circ}$ erreicht.

Das Aderlassfieber ist, soviel ich weiss, bis jetzt noch nicht studirt worden. Von vornherein könnte man eine Temperaturerniedrigung durch den Aderlass erwarten, und in den neuesten Monographien wird auch behauptet, dass die Blutentziehungen die Temperatur. herabsetzen.

Meine Versuche beweisen dagegen, dass sich eine stärkere Wärmebildung einstellt, wenn der Blutverlust gross war. Da ich die Blutentziehung durch Oeffnen der Carotis vornahm, so führe ich wohl mit Recht die erste Temperatursteigerung auf eine Reizung des Nervensystems durch Anämie zurück. Es wäre dies ein Analogon zu den Temperatursteigerungen infolge von psychischen Vorgängen, welche ich in meiner ersten Arbeit schon beschrieben habe.

Viel schwieriger ist es, die folgenden Temperaturerhöhungen und den fieberhaften Zustand zu erklären, da die Circulation darniederliegt und die 'Thiere infolge des stark verdinnten Blutes hochgradig anämisch sind. Ich wollte studiren, wie sich die Temperatur bei Hunden verhält, denen ich zuerst $2 \mathrm{~g}$ Antipyrin einspritzte und darauf einen reichlichen Aderlass beibrachte. Das Antipyrin hatte auf die erste Temperaturerhöhung keinen Einfluss, verminderte um ein $\mathrm{Ge}$ ringes den Anstieg der zweiten, doch nahm in der Folge die Curve wieder ibren aufsteigenden Verlauf an. In einigen Fällen brachten die Injectionen von Antipyrin bei Hunden mit Aderlassfieber eine Steigerung der Temperatur hervor. Ich habe schon erwähnt, dass sich nach den Gehirnläsionen infolge eines Aderlasses aufs Neue Temperatursteigerungen einstellen. Dort handelte es sich um Fälle, in denen die Temperatur nach der Gehirnverletzung in beständigem Abfallen begriffen war; jetzt könnte ich Fälle anführen, wo nach der Verletzung eine Temperatur von nahe $40^{\circ}$ bestand, und wo der Aderlass ein erneutes Steigen um $0,5-1^{0}$ zur Folge hatte. Erwähnen muss ich noch, dass der Aderlass bei tiefer Chloralisirung des Thieres keinen Einfluss auf dessen Temperatur mehr ausübt.

§ 11. Das Fieber dorch Staphylococcus pyogenes aureus und Wirkung der Medicamente auf dasselbe. Un mich so viel wie möglich den pathologischen Verhältnissen anzunähern, bediente ich mich zum Studium des Fiebers der Einspritzungen putrider Substanzen, jedoch haben die Hunde eine grosse Widerstandsfähigkeit gegen die fanligen Flüssigkeiten. Ich nahm daher zu den Reinculturen der eigentlichen 
Erzeuger von Fäulniss und Eiterung meine Zuflucht, unter denen sich der Staphylococens pyogenes aureus als besonders fiebererzeugend erwies.

Wenn man wenige Cubikcentimeter der fluissigen Gelatine, welche den Staphylococcus aureus enthält, in die Vena jugularis eines Hundes einspritzt, so beobachtet man zunächst eine leichte Erniedrigung der Mastdarmtemperatur um $0,5-1^{0}$, welche aber auch zuweilen fehlen kann; hierauf steigt die Temperatur allmählich, erreicht in 4-5 Stunden ihr Maximum, welches $2^{0}$ und mehr über der normalen liegt, und erhält sich mit unbedeutenden Schwankungen mehrere Tage auf dieser Höhe. Die Hunde, bei denen ich dieses Fieber hervorrief, starben sämmtlich. Einige verendeten nach 24 Stunden, andere starben nach 5-6 Tagen, und bei mehreren von diesen stellte sich am letzten Tage ein continuirliches Abfallen der Temperatur ein. Bei der Autopsie beobachtete ich immer eine leichte Röthung der Serosa in den Körperhöhlen; nur einmal fand ich einen grossen Lungenabscess, welcher vielleicht einer Embolie zu verdanken war, weil die injieirte Substanz nicht ganz flüssig war. In einem anderen Falle fand ich ein fibrinöses Coagulum in den Ventrikeln des Herzens. Ferner beobachtete ich, dass die Thiere in den letzten Stunden fast alle wie unter dem Einflusse lebhafter Schmerzen heulten. Die Darreichung von Antipyrin im Stadium des Temperaturanstiegs veranlasst einen Stillstand desselben und drückt zuweilen die Temperatur bis unter die Norm zuriick; diese antipyretische. Wirkung dauert je nach der Dosis 6-10 Stunden an.

Die bis jetzt geschilderten Gehirnläsionen bezogen sich sämmtlich auf Thiere, welche nicht fieberten, und es lag nahe, zu untersuchen, ob sich bei Vorhandensein des Fiebers durch eine Zerstörung derjenigen Gehirntheile, welche der muthmaassliche Sitz der Wärmecentren sind, eine Erniedrigung oder Erhöhung der Temperatur erzeugen lasse. Meine diesbezilglichen Versuche ergaben, dass weder eine Verminderung, noch auch eine starke Steigerung der 'Temperatur zu Stande kommt. Hieraus lässt sich demnach schliessen, dass der mittelst des Staphylococcus erzeugte fieberhafte Process nicht durch die Reizung eines Centrums zu Stande kommt, weil nach der Zerstörung desselben die Temperatur sich nicht erniedrigte, noch auch Lâhmung eines moderirenden Centrums, weil die Zerstörung des letzteren keine irgendwie bedentende Temperatursteigerung zur Folge hatte.

§ 12. Wirkung des Antipyrins auf die durch Cocain hervorgebrachten Temperaturerhöhungen. In meiner Arbeit über die physiologische 
Wirkung des Cocains ${ }^{2 b}$ ) habe ich gezeigt, dass unter allen bis jetzt bekannten pyrogenen Substanzen das Cocain diejenige ist, welche die raschesten und die stärksten Erhebungen der Körpertemperatur hervorbringt. $\mathrm{Da}$ ich wusste, dass die Temperatursteigerung nach der Anwendung von Cocain nicht von den Convulsionen herrührt, weil sie der Contraction der Muskeln vorangeht und weil sie auch beim curarisirten Thiere beobachtet wird, kam ich auf den Gedanken, mich des Cocains zur Erzeugung eines künstlichen Fiebers zu bedienen. Wenn man Hunden, die sich in vollkommen gutem Gesundheitszustand befinden, passende Dosen von Cocain injicirt, treten in weniger als einer $1 / 2$ Stunde die charakteristischen Fiebererscheinungen auf, nämlich Erhöhung der Temperatur und Beschleunigung des Pulses und der Respiration.

Wir wissen nicht, durch welchen Mechanismus das Cocain im Anfang seiner Wirkung diese Symptome, welche denen des Fiebers so ähnlich sind, zu erzeugen im Stande ist; sicher ist jedoch, dass die Vorgänge sehr complex sind, weil sich darauf schwere Störungen in der Innervation geltend machen, wie ich dies schon in meinen ersten Untersuehungen ïber das Cocain besehrieben habe.

Ich studirte den Einfluss des Antipyrins auf die durch Cocainum muriaticum zu Stande kommende Temperaturerhöhung in der $\mathrm{Ab}$ sicht, die Resultate von Girard und Sawadowski, welche nach der Zufügung von schweren Läsionen des Centralnervensystems den Versuchsthieren Antipyrin beibrachten, auf einem anderen Wege zu bestätigen. Meine Versuche hatten das Resultat, dass das Antipyrin auf derartige Erhöhungen gar keinen Einfluss ausübt. Wenn man das Cocain vor oder kurz nach der Einfüthrung des Antipyrins injicirt, so wird die Vermehrung der Temperatur nicht alterirt. Um mich von der Richtigkeit dieser Thatsache zu überzeugen, variirte ich die Dosen des Medicaments und die Art und Weise der Darreichung, indem ich bei jedem Hunde Sorge trug, zunächst den Verlauf der Temperaturcurve bei einer bestimmten Cocaingabe festzustellen. Es genügten diese Versuche, um zu beweisen, dass die Wirkung des Antipyrins nicht, wie Sawadowski meint, gegen den Theil des Nervensystems gerichtet ist, von dem die Temperatur abhängt, weil trotz der Darreichung starker Antipyrindosen die vermehrte Wärmebildung andauert. Dies ist zwar nur ein indirecter Beweis, aber ich halte ihn darum für nicht minder bedeutsam.

$\S 13$. Ist das Fieber ein nervöses Phänomen? Die fieberhaften Vorgänge zeigen unter einander eine grosse Verschiedenheit in ihrem Typus, in den Entstehungsursachen und in ihrem Verhalten zu der Wirkung der Arzneimittel. Es ist nicht möglich, von einer rationellen Fiebertherapie zu sprechen, so lange uns die wahre Natur der" verschiedenen fieberhaften Processe verborgen bleibt. 
Im Jahre 1886 studirte ich ${ }^{2 a}$ ) das Verhalten mehrerer Substanzen, welche durch Einwirkung anf das Nervensystem die Körpertemperatur erhöhen oder herabsetzen, und kam dabei zu folgenden Resultaten:

1. Cocain und Strychnin haben nach Anwendung grosser Dosen von Chloralhydrat auf die Temperatur keinen Einfluss mehr. 2. Das Curare, welches auf die Muskeln lähmend wirkt, hat dessenungeachtet eine schwache Einwirkung auf die Temperatur. 3. Das Chloral vermindert die Temperatur bedeutend stärker als das Curare, obwohl es gar nieht auf die Muskeln wirkt.

Auf Grund dieser Beobachtungen hatte ich angenommen, dass gewisse Substanzen, wie das Chloral, den thermisehen Einfluss des Nervensystems lähmen, andere dagegen, wie das Curare in kleinen Dosen, demselben keinen Eintrag thun.

Ich habe nunmehr dieselben Versuche an dem mit Staphylococcus aureus erzeugten Fieber wiederholt, und aus meinen Experimenten geht Folgendes hervor:

a) Die Injection des Staphylococcus brachte bei einem chloralisirten Hund, dessen Temperatur in stetigem Absinken begriffen war, eine beträchtliche Temperatursteigerung hervor. b) Bringt man dem Thiere, nachdem das Staphylococcusfieber eine bedeutende Höhe erreicht hat, Chloral bei, so beobachtet man eine Verminderung der Temperatur, welche jedoch bald wieder einem Ansteigen Platz macht. c) Bei grösseren Dosen Chloralhydrat ist die Herabsetzung der Temperatur noch ausgesprochener, aber das Fieber nimmt danach seinen ansteigenden Verlauf wieder an und erreicht bald die frühere Höhe.

Die Unsicherheit, welche gegenwärtig in der Physiologie in Bezug auf die Quellen der thierischen Wärme herrscht, spiegelt sich auch in der Pathologie und in der Therapie wieder. So kommt es, dass heute viele Pathologen das einfache Fieber anf eine Krankheit der thermischen Centren zurückführen, und dass trotzdem Niemand über den Sitz dieser Centren Aufschluss geben kann. Ich hoffe den Beweis geliefert zu haben, dass für die Annahme von Centren im Gehirn, von denen die Erhöhung der Körpertemperatur abhängt, die thatsächliche Grundlage fehlt; es bleibt mir jetzt nur noch übrig, das Rückenmark und die übrigen Theile des Nervensystems einer Prüifong zu unterziehen.

Meine ersten Untersuchungen, welche eine Art Einleitung zu dieser Studie iiber das Fieber bilden, waren darauf gerichtet, den Einfluss des Nervensystems auf die Körpertemperatur kennen zu lernen. Da meine Anschauungen mit denen angesehener Experimentatoren, von welchen ich nur Chauveau ${ }^{23}$ ) zu erwähnen brauche, der auch in den Muskeln die wichtigste Quelle der thierischen Wärme sucht, nicht tibereinstimmen, so 
muss ich die von mir erhaltenen Resultate kurz andenten. Meine Versuche haben mich tuberzeugt, dass die Eigenschaft, Wärme zu produciren, überhaupt keinem Gewebe ausschliesslich zugeschrieben werden darf, sondern dass alle lebenden Zellen Wärme erzeugen. Die Thatsachen, welehe ich kurz darlegen will, dienen mir als Grundlage für die Annahme, dass es ein Fieber rein nervöser Natur giebt, und dass die Muskeln in der Wärmeproduction keine Ausnahmestellung einnehmen.

1. Die Vermehrung der Wärme, welche bei der Muskelcontraction eintritt, ist eine Begleiterscheinung und ist nicht nothwendig zum $\mathrm{Zu}$ standekommen der Zuckung. 2. Diese Vermehrung der Wärme hält nach dem Aufhören der Contraction nicht inne, sondern dauert noch einige Zeit an. 3. Die Erregung der sensiblen Nerven ruft auf dem Reflexwege eine noch grössere Wärmeproduction hervor. 4. Die Körpertemperatur hält mit der geleisteten Muskelarbeit nicht gleichen Schritt. 5. Eine andauernde ermüdende Muskelarbeit genügt nicht, un die Temperatur in einem Körpertheile iiber der Norm zu erhalten. 6. Die Contraction der Muskeln bringt keine solche Temperaturerhöhung zu Stande, wie sie die Nerventhätigkeit bei psychischen Vorgängen für sich allein zu erzeugen vermag. 7. Bei der Strychninvergiftung hängt die Temperaturerhöhung weniger von den tetanischen Contractionen, als von der erhöhten Erregbarkeit des Nervénsystems ab. 8. Die Erregungen und psychischen Vorgänge bringen eine Vermehrung der Temperatur hervor, ohne dass dabei eine grössere Thätigkeit der Muskeln besteht. 9. Das Strychnin kann die Temperatursteigerung trotz der durch Curare erzeugten absoluten Unbeweglichkeit des Thieres hervorbringen.

Ueber diesen letzten Punkt sehe ich mich genöthigt, einige Erklärungen abzugeben. C. Richet und Langlois machten zusammen Versuche iiber die Wirkung des Strychnins und des Cocains an curarisirten Thieren und sahen keine Temperatursteigerung mehr eintreten, wenn das Thier vollständig gelähmt war. Ich glaube, dass dieses Ergebniss darauf zurückzuführen ist, dass die genannten Autoren zu grosse Dosen Curare anwandten, woriber ich mich schon früher ausgesprochen habe.

Thatsächlich sagt E. Reichert ${ }^{24}$ ) in seiner Arbeit iiber die Wirkung des Cocains auf die Functionen der thierischen Wärme, dass er meine Beobachtung vollständig bestätigen konnte, und führt auf S. 442 (l. c.) 2 Versuche an, aus denen hervorgeht, dass seine Resultate mit den meinigen identisch sind.

Wenn ich meine Beobachtungen iiber das Fieber noch einmal überblicke, so glaube ich behaupten zu können, dass sich die Körpertemperatur auf zweierlei Weise vermehren kann: die eine häng rom Nervensystem ab, die andere ist vom Nervensystem unabhängig. Diese beiden verschiedenen Fiebergattungen sind in meinen Versuchen durch zwei verschiedene Gruppen repräsentirt: die Temperaturerhöhungen, welche nach schweren Blutverlusten und nach der Anwendung von Cocain beobachtet werden, bilden die eine Gruppe der vom Nervensystem abhängenden, die nach Injection des 
Staphylococcus aureus zu Stande kommenden Temperatursteigerungen sind unabhängig vom Nervensystem und entsprechen dem eigentlichen, von den Geweben ausgehenden Fieber: mit anderen Worten, das Fieber hat im ersten Falle einen centralen Ursprung; weil es vom Nervensystem abbängt, im zweiten Fall, beim Staphylococcusfieber, greift die Ursache peripher an, und hat ihren Sitz in den Geweben. Diese zweite Art hier in Frage kommenden Fiebers war schon durch die Untersuchungen von A. Murri ${ }^{25}$ ) bekannt, und meine Versuche mit dem Staphylococcus haben die Experimente von Murri durch eine andere Methode bestätigt; obgleich anch in diesem Falle die Wirkung des Nervensystems sich nicht ganz ausschliessen lässt, da wir ja bis jetzt noch nicht wissen, in welcher Weise die Nervenendigungen in den Geweben durch die pyrogenen Agentien beeinflusst werden. Ich kann mir auf keine andere Weise die Thatsache erklären, dass das Chloralhydrat die charakteristische Wärmeentwicklung nach $\mathrm{Co}$ caingebrauch verhindert, während es auf das durch Staphylococcus erzeugte Fieber keinen Einfluss auszuiuben vermag. Da ich nicht zugeben kann, dass der Staphylococcus intensiver wirkt, als das Coeain, weil das letztere sogar viel bedeutendere Temperatursteigerungen erzeugt, als das Cocain, so muss ich annehmen, dass es sich hier um zwei vollkommen verschiedene Arten der Wärmebildung handelt, von denen die eine vom centralen Nervensystem abhängt, die andere davon unabhängig ist.

\section{Literaturverzeichniss.}

1) C. Golg i, Beitrăge zur path. Anat. u. allg. Pathol. IV. Bd. S. 433.

2) U. Mosso, a) Virchow's Archiv f. path. Anat. u. Physiol. CVI. Bd. S. 80. - b) Dieses Archiv. XXIII. Bd. S. 153.

3) J. Tscheschichin, Archiv für Anat. u. Physiol. 1866. S. 151.

4) P. Lewizky, Virchow's Archiv. 1869. XLVII. Bd. S. 352.

5) L. Bruck u. A. Günter, Pflüger's Archiv 1870. III. Bd. S. 578.

6) J. Schreiber, Ebenda 1874. VIII. Bd. S. 576.

i) Н. C. Wood, Fever, Phyladelphia 1880.

8) Ch. Richet, a) Comptes rendus, 31. März 1884. p. 827. - b) Ibidem. 1. sem. 1885. p. 1021. - c) Archives de Physiologie 1885. p. 495.

9) Isaac 0 tt, Journal of nervous and mental diseases. 1884. Vol. XI.

10) A ronsohn u. Sachs, Pfluger's Archiv 1885. XXXVIr. Bd. S. 232.

11) H. Girard, Archives de Physiologie 1886. S. 281:

12) Isaac O tt, a) Journal of nervous and mental diseases. März 1887. - b) Ibid. Fehr. 1888.

13) Girard, Archives de Physiologie 1888. p. 312. 
340 XIX. Mosso, Die Lehre vom Fieber in Bezug auf die cerebralen Wärmecentren.

14) Angelo Mosso, Sur une nouvelle méthode pour écrire les mounements des vaisseaux sanguins chez l'homme. Comptes Rendus vol. LXXXII. p. 282.

15) D'Arsonval, Journal de l'Anatomie 1886. p. 114.

16) Ch. Richet, Archives de Physiologie 1885. p. 460.

17) Rosenthal, Archiv f. Anat. und Physiol. 1887 u. 1888 . S. 349.

18) Langlois, Journal de l'Anatomie et de la Physiologie 1887. p. 401.

19) Fredericq, Mémoires de l'Acad. roy. de médecine de Belgique. Vol. VIII.

20) Maragliano, Archivio italiano di clinica medica. 1888.

21) Gir ard, Revue medicale de la Suisse Romande. 1887.

22) Sawadowski, Centralblatt f. med. Wiss. Nr. 8, 9, 10. 1888.

23) Chauveau, Revue Scientifique 1888.

24) Reichert, University medical magazin. Pennsilvania. Mai 1889. p. 439.

25) A. Murri, Riforma medica. No. 249. 1888. 